\title{
Experiences of magnetic resonance imaging scan- ning in patients with pacemakers or implantable cardioverter-defibrillators
}

\author{
Donghoon Han, Si-Hyuck Kang, Youngjin Cho, and Il-Young Oh
}

Department of Internal Medicine, Seoul National University Bundang Hospital, Seongnam, Korea

Received: July 19, 2017

Revised : August 9, 2017

Accepted: August 23, 2017

\section{Correspondence to}

\section{Il-Young Oh, M.D.}

Division of Cardiology, Department of Internal Medicine, Seoul National University Bundang Hospital, 82 Gumi-ro 173beongil, Bundang-gu, Seongnam 13620 , Korea

Tel: $+82-31-787-7056$

Fax: +82-31-787-4290

E-mail: ilyoung.oh@snubh.org
Background/Aims: Despite the U.S. Food and Drug Adminstration approving a magnetic resonance imaging (MRI)-conditional pacemaker system in 2011, many physicians remain reluctant to perform MRI scanning in patients with cardiac implantable electronic devices. Herein, we aimed to evaluate the real-world safety of MRI in these patients.

Methods: This single-center retrospective study examined the interrogation data and outcomes of patients with pacemakers or implantable cardioverter defibrillators who underwent MRI. MRI interrogation data were collected pre- and post-MRI and after 1 month of follow-up; these included the lead impedance, measured P- and R-wave amplitudes, and capture threshold. We compared these results between the magnetic resonance (MR)-conditional and conventional groups.

Results: From September 2013 to December 2015, 35 patients with cardiac implantable electronic devices underwent 43 MRI scans, with a mean follow-up of 5 months. Among these 35 patients, 14 (40\%) had MR-conditional devices and 21 $(60 \%)$ had conventional devices. Seven patients had high voltage devices, which were all the conventional type. There were no adverse events associated with MRI during the follow-up period, and there were no significant differences in the interrogation data changes between the conventional and MR-conditional groups.

Conclusions: This single-center retrospective study found that MRI can be performed safely in patients with pacemakers or implantable cardioverter defibrillators, regardless of the MRI support, as long as appropriate precautions are taken.

Keywords: Pacemakers; Defibrillators, implantable; Magnetic resonance imaging; Interrogation data; Safety

\section{INTRODUCTION}

Patients with cardiac implantable electronic devices (CIEDs) are increasing, and these patients usually have multiple comorbidities that require medical attention $[1,2]$. A previous study estimated that these patients have an approximately $75 \%$ likelihood of being indicated for a magnetic resonance imaging (MRI) scan over the lifetime of the device [3]. However, patients with CIEDs may experience some adverse effects induced by MRI, including tissue heating, capture failure, increasing impedance, and lead dislocation, among others [4-7]. Further, although there is no way to directly measure tissue heating, this may cause tissue scars, which in turn may 
induce changes in the impedance and threshold values [8-10].

Recently, magnetic resonance (MR)-conditional pacemakers and lead systems have been developed to address these issues. Such pioneering technologies can be safely used under specific conditions, including a specific field magnitude of 1.5 Tesla (T), exclusion body zones, and specific absorption rate limits of $<2 \mathrm{~W} / \mathrm{kg}$. Recent European guidelines have stated that MRI at 1.5 $\mathrm{T}$ can be performed safely following the manufacturer instructions in patients with an MR-conditional system (class IIa, level of evidence B) [11].

However, many physicians still remain reluctant to perform MRI scans in patients with CIEDs, even in cases of MR-conditional systems [12]. The Really ProMRI registry is an ongoing study assessing the annual rate of MR examinations in patients with MR-conditional implants, including both pacemakers and implantable cardioverter defibrillators (ICDs), and which aims to detect the main factors limiting MRI in these patients [13]. Herein, in order to determine the safety of MRI scanning in patients with pacemakers or ICDs in real clinical practice, we reviewed our experiences of MRI scanning in patients with pacemakers or ICDs.

\section{METHODS}

\section{Study population}

Thirty-five consecutive patients with pacemakers or ICDs who underwent MRI scans between September 2013 and December 2015 were retrospectively reviewed. In all cases, the MRI scan was ordered by the patient's treating physician. Before the MRI scan, the physician and consultant cardiologist determined that the potential benefit of the diagnostic data obtained by MRI significantly outweighed the potential risk for device failure. To evaluate the safety of MRI scans in patients with all kinds of pacemakers or ICDs, all consecutive patients were enrolled, and patients with conventional devices were not excluded. However, two patients were excluded because they had undergone MRI without a cardiology consult.

The Institutional Review Board Committee of Seoul National University Bundang Hospital approved this retrospective study (B-1612-375-102) and waived the need for informed consent for the access to electric medical records. The study was performed in accordance with the tenets of the Declaration of Helsinki.

\section{MR-conditional vs. conventional groups}

The patients were divided into two groups: the MR-conditional and conventional groups. The patients were classified into the MR-conditional group if the following two factors were satisfied: (1) MR-conditional leads and (2) MR-conditional generators. If at least one of these was not satisfied, they were classified into the conventional group.

\section{MRI scans and device interrogation}

Before each MRI scan, the pacemakers were reprogrammed to an asynchronous pacing mode or MRI mode. For patients with ICDs, the mode of tachyarrhythmia therapies was disabled. The patients were monitored throughout the MRI with continuous electrocardiogram recording and a pulse oximeter. A cardiologist with training in how to place and use a temporary external cardiac pacemaker was present throughout the MRI study.

Device interrogation was performed before and after the MRI study. The parameters interrogated and recorded included the battery voltage, pacing thresholds, $\mathrm{P}$-wave and R-wave amplitudes, pacing lead impedances, and high-voltage lead impedance.

\section{Statistical analysis}

Statistical analysis was performed with dbSTAT version 5 (dbSTAT, Seoul, Korea) and SPSS version 22 (IBM Co., Armonk, NY, USA). The chi-square test, Wilcoxon's rank test, Kruskal-Wallis test, and analysis of variance were used to compare the data between the groups. A $p<0.05$ was considered statistically significant.

\section{RESULTS}

\section{Patients}

The 35 patients with pacemakers or ICDs underwent 43 MRI scans. The mean age of the patients was 70.1 years (range, 42 to 90), and 19 (55.9\%) were male. There were no differences between the MR-conditional and conventional groups in any of the baseline characteristics (Ta- 
Table 1. Clinicodemographic characteristics of the study patients

\begin{tabular}{|c|c|c|c|c|}
\hline Characteristic & $\operatorname{All}(\mathrm{n}=35)$ & MR-conditional $(n=14)$ & Conventional $(\mathrm{n}=21)$ & $p$ value \\
\hline Age, yr & $70.1 \pm 11.4$ & $70.5 \pm 9.8$ & $69.8 \pm 12.5$ & 0.85 \\
\hline Male sex & $19(54 \cdot 3)$ & $7(50.0)$ & $12(57.1)$ & 0.55 \\
\hline \multicolumn{5}{|l|}{ Comorbidities } \\
\hline Hypertension & $19(54.2)$ & $5(35 \cdot 7)$ & $14(66.7)$ & 0.05 \\
\hline Diabetes mellitus & $13(37.1)$ & $5(35 \cdot 7)$ & $8(38.1)$ & 0.58 \\
\hline Atrial fibrillation & $14(40.0)$ & $4(28.6)$ & $10(47.6)$ & 0.21 \\
\hline Cerebral infarction & $8(22.9)$ & $3(21.4)$ & $5(23.8)$ & 0.79 \\
\hline \multicolumn{5}{|l|}{ CIED indication } \\
\hline Pacemaker & $28(80.0)$ & $14(100)$ & $14(66.7)$ & 0.01 \\
\hline SSS & 15 & 6 & 9 & \\
\hline CAVB & 13 & 8 & 5 & \\
\hline ICD & $7(20.0)$ & o & $7(33 \cdot 3)$ & - \\
\hline Primary prevention & 5 & o & 5 & \\
\hline Secondary prevention & 2 & o & 2 & \\
\hline \multicolumn{5}{|l|}{ No. of MRI scans } \\
\hline 1 & $29(82.9)$ & $13(92.9)$ & $16(76.2)$ & 0.32 \\
\hline$\geq 2$ & $6(17.1)$ & $1(7.1)$ & $5(23.8)$ & 0.23 \\
\hline
\end{tabular}

Values are presented as mean \pm SD or number (\%).

MR, magnetic resonance; CIED, cardiac implantable electronic device; SSS, sick sinus syndrome; CAVB, complete atrioventricular block; ICD, implantable cardioverter defibrillator; MRI, magnetic resonance imaging.

ble 1). The details of the device models and MRI scans are described in Supplementary Table 1. Twenty-eight patients had a pacemaker (80.0\%) and seven had ICDs (20.0\%). All patients had undergone device implantation between 2003 and 2014, and one underwent generator revision in 2008. Six patients underwent MRI scanning more than two times (four patients: 2 times; one patient: 3 times; one patient: 4 times).

For the analyses, 14 and 21 patients were classified into the MR-conditional and conventional groups, respectively. Among the 21 patients in the conventional group, 11, one, and nine patients had only MR-conditional leads, only MR-conditional generators, and both conventional leads and generators, respectively.

\section{MRI scans}

Among the 43 MRI scans, 16 (37.2\%) and 27 (62.8\%) scans were performed in the MR-conditional and conventional groups, respectively. Twenty-five (58.1\%), 15 (34.9\%), two (4.7\%), and one (2.3\%) of the 43 MRI scans were performed for imaging of the head, spine, abdomen or pelvis, and lower extremities, respectively. All MRIs were performed using a 1.5-T magnet (Table 2).

Forty-one MRIs were performed with the MRI mode or asynchronous pacing mode (e.g., ventricular asynchronous pacing [VOO] or dual-chamber asynchronous pacing [DOO]), and two MRIs were performed without any mode change. Of these, one was performed in the MR-conditional group and one in the conventional group (MR-conditional leads only). No patients experienced MR-related complications such as tissue heating, failure of capture, increasing impedance, or lead dislocation.

\section{Follow-up}

The mean follow-up of device interrogation was 5.4 months (range, 0.2 to 13.8). During the follow-up period, no generator/lead failure or battery problems occurred. Eight patients did not visit the CIED outpatient clinic after their MRI scan. Among these eight patients, three had died due to diseases not related to the devices, such as brain tumor, respiratory arrest, and sepsis following aspiration pneumonia; four were alive without specific complications associated with the devices; and one was 
Table 2. Differences in the MRI modalities used between the MR-conditional and conventional groups

\begin{tabular}{|c|c|c|c|c|}
\hline Variable & All $(n=43)$ & $\begin{array}{l}\text { MR-conditional } \\
\qquad(\mathrm{n}=16)\end{array}$ & $\begin{array}{l}\text { Conventional } \\
\qquad(\mathrm{n}=27)\end{array}$ & $p$ value \\
\hline \multicolumn{5}{|l|}{ MRI location } \\
\hline Brain & $25(58.1)$ & 11 & 14 & 0.29 \\
\hline Spine & $15(34 \cdot 9)$ & 5 & 10 & 0.71 \\
\hline Abdomen \& pelvis & $2(4.7)$ & 0 & 2 & \\
\hline Extremities & $1(2.3)$ & $\mathrm{o}$ & 1 & \\
\hline \multicolumn{5}{|l|}{ MRI mode } \\
\hline $\begin{array}{l}\text { MRI mode or } \\
\text { asynchronous pacing mode (e.g., VOO or DOO) }\end{array}$ & $41(95 \cdot 3)$ & 15 & 26 & 0.71 \\
\hline Without mode change & $2(4 \cdot 7)$ & 1 & 1 & 0.70 \\
\hline \multicolumn{5}{|l|}{ CIED } \\
\hline Pacemaker & $32(74.4)$ & 16 & 15 & 0.01 \\
\hline ICD & $11(25.6)$ & o & 11 & \\
\hline
\end{tabular}

Values are presented as number (\%).

MRI, magnetic resonance imaging; MR, magnetic resonance; VOO, ventricular asynchronous pacing; DOO, dual-chamber asynchronous pacing; CIED, cardiac implantable electronic devices; ICD, implantable cardioverter defibrillator.

lost to follow-up.

\section{Mean lead impedance, sensing, and capture threshold}

The differences in lead impedance, sensing, and capture threshold between pre-MRI, post-MRI, and follow-up were analyzed. As a result, there were no statistically significant differences over time (Table 2, Fig. 1). Moreover, we analyzed the differences in the mean values in lead impedance, sensing, and capture threshold at pre-MRI, post-MRI, and follow-up between the MR-conditional and conventional groups (Supplementary Table 3, Fig. 2). Similarly, there were no significant differences in any of these values between the two groups in each period, except for in the atrial lead threshold. The mean atrial threshold of the conventional group was higher than that of the MR-conditional group at the follow-up $(p=0.02)$. However, there was no significant difference between the two groups in the post-MRI threshold ( $p=$ o.89).

\section{Changes in lead impedance, sensing, and capture threshold}

The changes between pre- and post-MRI and between pre-MRI and the follow-up in the impedance, sensing, and captured threshold were analyzed in the MR-con- ditional and conventional groups (Table 3). There were no significant differences between the two groups in the changes in atrial and ventricular lead impedance, sensing, and capture threshold, despite the mean atrial threshold of the conventional group being higher than that in the MR-conditional group at the follow-up.

\section{ICD devices}

We measured the high voltage impedance in the seven patents with ICDs. There were no significant differences in high voltage impedance among the pre-MRI, postMRI, and follow-up periods $(p=0.79)$ (Supplementary Fig. 1). Among these seven patients, no adverse event occurred during the MRI scan or follow-up period. After the MRI scan, one patient experienced ventricular fibrillation episodes, which were detected and terminated successfully.

\section{DISCUSSION}

In this single-center retrospective review of patients with pacemakers or ICDs undergoing MRI, we found that these patients could undergo MRI scans without any adverse events. When comparing the MR-condi- 

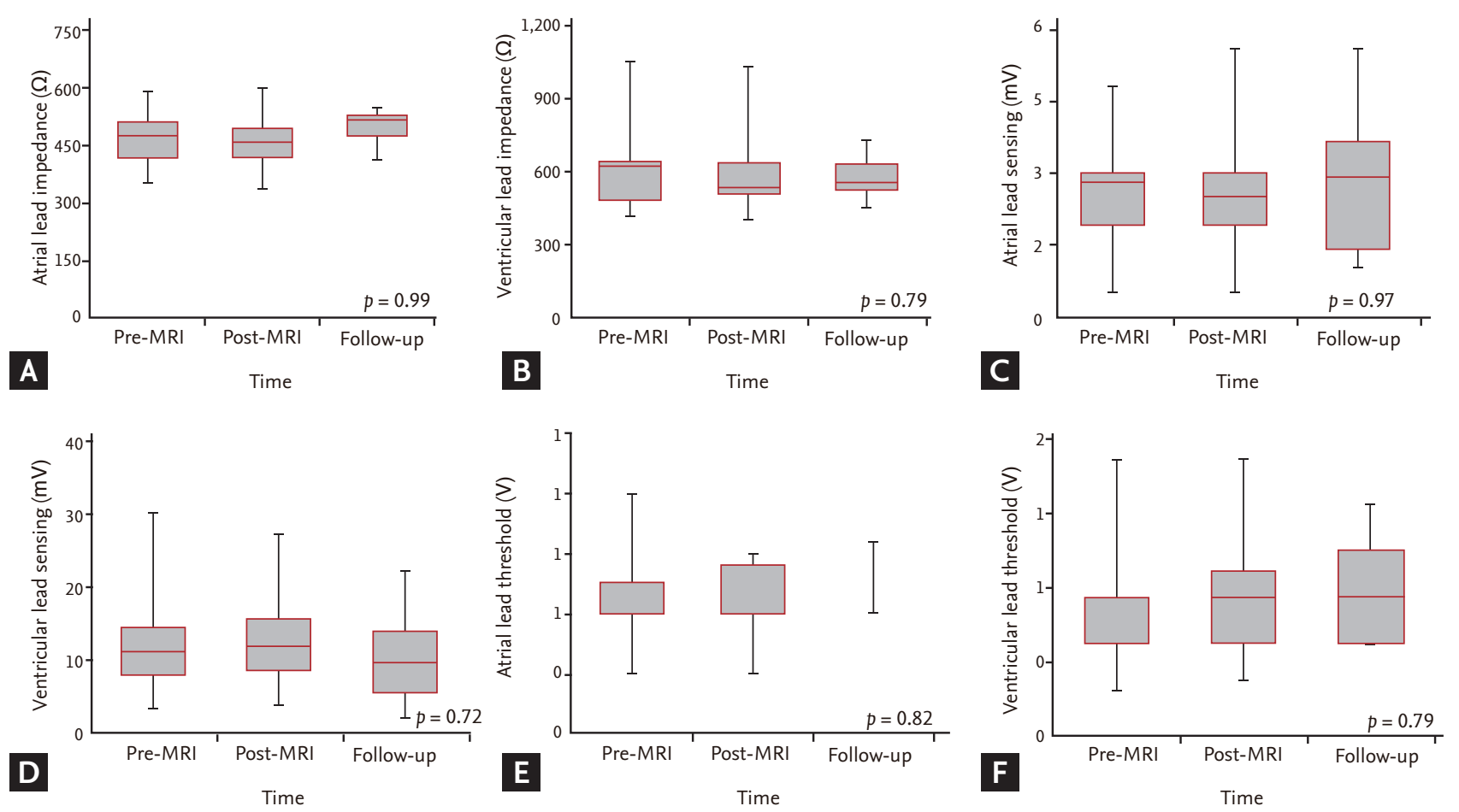

Figure 1. Changes in the (A, B) atrial and ventricular impedance, (C, D) lead sensing, and (E, F) captured threshold among pre-/ post-magnetic resonance imaging (MRI) and follow-up.

tional and conventional groups, there were no significant differences in the lead impedance, sensing, and capture threshold.

The safety of MRI scans for patients with CIEDs is still debated, despite previous studies having concluded that there were no significant differences in the safety and adverse events in these patients $[3,12,14-20]$. In our real-world practice, 35 patients with pacemakers or ICDs underwent 43 MRI scans without any adverse events. We experienced no device-related complications, including device-related symptoms, damage to the hardware, inhibition of pacing, inappropriate programming, or device-related arrhythmia. All MRI scans were performed following the previously validated safety protocol reported by Nazarian et al. [14], except 2 scans. The mean values of the lead impedance, sensing, and capture threshold did not differ among the pre-MRI, post-MRI, and follow-up periods. To rule out the effect of MR-conditional devices, we compared these factors between the MR-conditional and conventional groups. The atrial captured thresholds during the pre-MRI and follow-up periods were higher in the conventional group than in the MR-conditional group. However, in both groups, the changes between the pre-MRI and post-MRI and between the pre-MRI and follow-up periods did not differ. No other significant difference was observed between the two groups.

In our study, we included seven patients with conventional ICDs, who safely underwent 11 MRI scans. Of these, one patient experienced 10 episodes of spontaneous ventricular tachycardia/ventricular fibrillation and nine therapeutic shocks after MRI (Supplementary Fig. 2 briefly summarizes the patient's clinical history, symptoms, and signs). The patient had an ICD due to ischemic cardiomyopathy, and had already experienced one episode of therapeutic shock in 2012. Three years later, he underwent MRI to evaluate the extent of a coccyx sore. Although he experienced nine therapeutic shocks after the MRI scan, all episodes of ventricular tachycardia/ventricular fibrillation were detected properly and all therapeutic shocks were appropriate. Therefore, we regarded that all ICDs were working properly after the MRI scans, and we consider that patients with conventional ICDs can undergo MRI safely as long as 


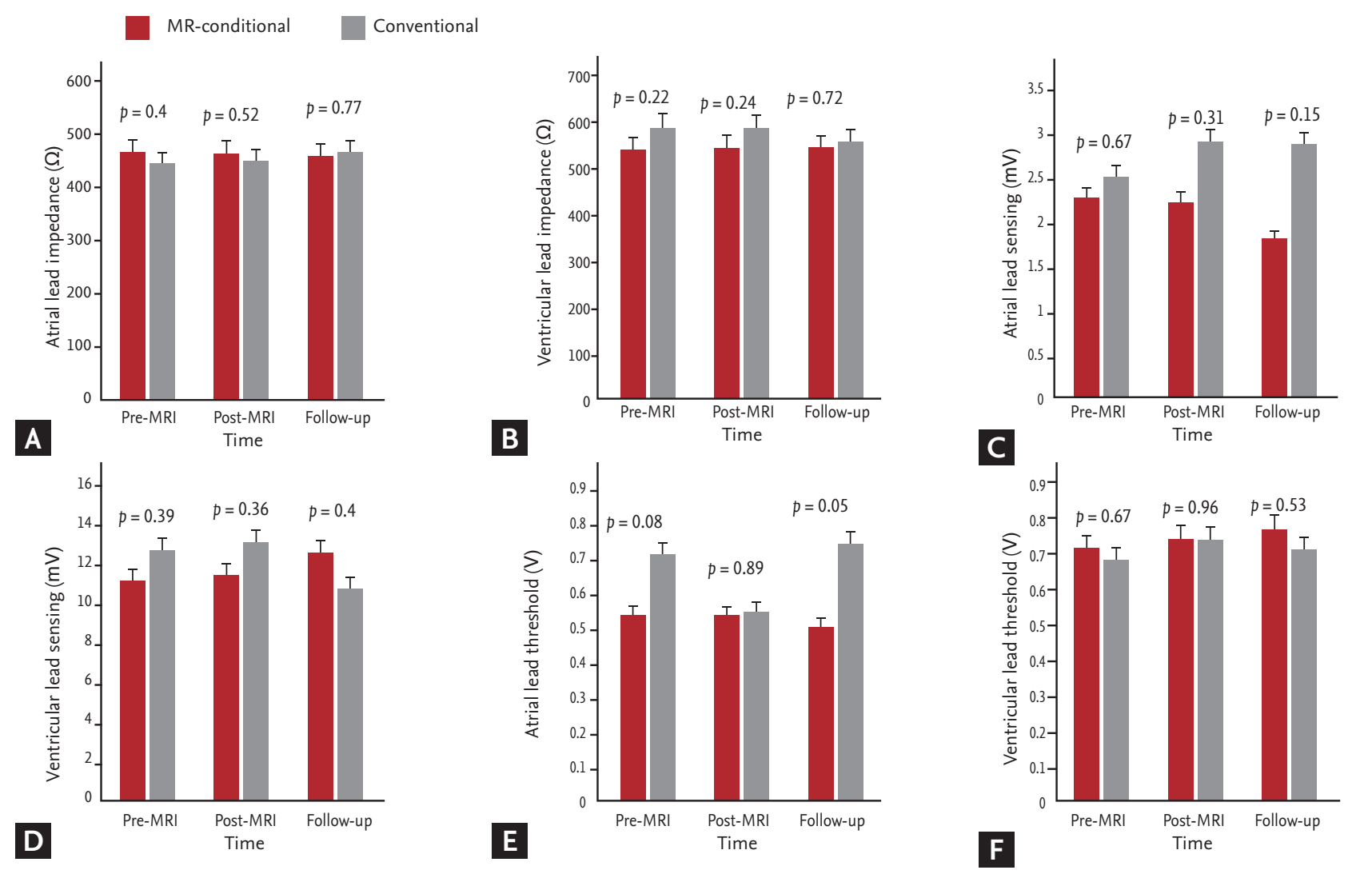

Figure 2. Changes in the (A, B) atrial and ventricular impedance, (C, D) lead sensing, and (E, F) captured threshold between the magnetic resonance (MR)-conditional and conventional groups at pre-/post-magnetic resonance imaging (MRI) and follow-up.

they are carefully selected and a strict protocol is utilized. Furthermore, MR-conditional ICDs have recently been developed and approved by the U.S. Food and Drug Adminstration (FDA) [21]. Therefore, it is necessary to compare the outcomes between MR-conditional and conventional ICDs to establish a standardized safety protocol.

Nazarian et al. [14] enrolled patients with pacemakers and ICD generators manufactured after 2000 and reported that patients with the selected CIEDs were able to undergo MRI scans safely. This finding is very similar to our results. All patients enrolled in our study underwent device implantation after 2003, with the leads and generators manufactured in 2004 or later (Supplementary Table 4). These results suggest that recently manufactured devices may be safe for MRI scans.

Recently, the safety of new MR-conditional pacing systems was reported [18], and MR-conditional CIEDs, from various manufacturers, have been used worldwide since they achieved FDA approval in 2011. Nevertheless, MRI scanning in patients with CIEDs remains a controversial issue, and the evidence supporting the current guidelines is still weak (IIb B or IIa B) [22]. Hence, many clinicians hesitate to order MRI for patients with CIEDs. Accordingly, in the present study, we aimed to assess which changes, if any, occur in the patients' devices before and after MRI. However, our study showed that there were no adverse events or significant changes in any device parameter in both the MR-conditional and conventional groups. This finding is very similar to those of previous studies on the topic $[14,15,17,18,23-26]$. Recently, Russo et al. [25] reported that there was no device or lead failure after nonthoracic MRI with $1.5 \mathrm{~T}$ in patients with non-MRI-conditional pacemakers or defibrillators. They also enrolled patients with pacemakers or implantable cardioverter-defibrillators implanted after 2001. However, their study differed from ours in several different points. First, Russo et al. [25] set death 
Table 3. Differences in the atrial and ventricular impedance, sensing, and captured threshold at pre-/post-MRI and FU between the MR-conditional and conventional groups

\begin{tabular}{|c|c|c|c|}
\hline Variable & MR-conditional $(n=16)$ & Conventional $(\mathrm{n}=27)$ & $p$ value \\
\hline \multicolumn{4}{|l|}{ Impedance, $\Omega$} \\
\hline \multicolumn{4}{|l|}{ Atrial } \\
\hline Post change & $-2.1 \pm 27.4$ & $-4.0 \pm 16.1$ & 0.86 \\
\hline FU change & $-6.9 \pm 33.26$ & $-12.5 \pm 22.2$ & 0.76 \\
\hline \multicolumn{4}{|l|}{ Ventricular } \\
\hline Post change & $-4 \cdot 3 \pm 18.2$ & $4.0 \pm 29.2$ & 0.33 \\
\hline FU change & $-8.3 \pm 38.29$ & $-10.3 \pm 31.9$ & 0.88 \\
\hline \multicolumn{4}{|l|}{ Sensing, mV } \\
\hline \multicolumn{4}{|l|}{ Atrial } \\
\hline Post change & $-0.15 \pm 0.26$ & $0.38 \pm 1.25$ & 0.15 \\
\hline FU change & $-0.39 \pm 0.99$ & $-0.28 \pm 0.53$ & 0.83 \\
\hline \multicolumn{4}{|l|}{ Ventricular } \\
\hline Post change & $0.31 \pm 0.88$ & $0.39 \pm 2.7$ & 0.91 \\
\hline FU change & $0.19 \pm 5.27$ & $-1.69 \pm 7.99$ & 0.51 \\
\hline \multicolumn{4}{|c|}{ Capture threshold, V } \\
\hline \multicolumn{4}{|l|}{ Atrial } \\
\hline Post change & $0 \pm 0.11$ & $-0.1 \pm 0.13$ & 0.11 \\
\hline FU change & $-0.03 \pm 0.05$ & $0.03 \pm 0.16$ & 0.32 \\
\hline \multicolumn{4}{|l|}{ Ventricular } \\
\hline Post change & $0.09 \pm 0.29$ & $0.03 \pm 0.11$ & 0.46 \\
\hline FU change & $0.07 \pm 0.23$ & $0.02 \pm 0.15$ & 0.52 \\
\hline
\end{tabular}

Values are presented as mean $\pm \mathrm{SD}$.

MRI, magnetic resonance imaging; FU, follow-up; MR, magnetic resonance.

and device or lead failure as the primary endpoints and focused on the changes in battery voltage, lead threshold, impedance, and decreasing P- and R-wave amplitudes. In contrast, we focused on the changes in the lead profile, including changes in the absolute values of impedance, lead sensing, and captured threshold. Second, our retrospective study included some patients with a follow-up period of more than 1 year (maximum follow-up, 420 days). As a result, we found that the devices remained stable more than 1 year after MRI. In addition, we also analyzed the interrogation data of the generators and leads 1 month after MRI to evaluate any subacute complications.

Our report has some limitations. First, the number of patients was small, even though we enrolled all patients with pacemakers or ICDs who underwent MRI during the study period. For this reason, it was difficult to compare the values of each patient. Although we tried to overcome this limitation by analyzing the changes in all data and by comparing the differences between the MR-conditional and conventional groups, it also suggests that many clinicians are still reluctant to perform MRI in patients with CIEDs. Second, as a result of the retrospective nature of the study, we could assess only the function of the devices, which indirectly reflects potential tissue damage. For the same reason, the follow-up periods were also uneven. However, interrogations after MRI were acquired in all patients, and patients who did not visit an outpatient clinic were followed up by telephone. Nonetheless, despite the small number of cases, our retrospective survey conducted at a single center included diverse cases reflecting the real-world practice, including seven patients with conventional high-voltage devices and six patients who underwent MR scanning 
more than 2 times, although the interval periods were variable, ranging from 7 days to more than 1 year. Furthermore, two patients underwent the MRI scan without mode changes due to missing the screening test. In all of these cases, we found that the devices used were stable and safe after MRI.

In conclusion, MRI may be performed safely in patients with MR-conditional or conventional pacemakers or ICDs if a strict protocol based on device selection is used and careful monitoring is conducted. These results may help support the current guidelines and could provide evidence to guide further research.

\section{KEY MESSAGE}

1. Our single-center study demonstrated the safety of pacemakers or implantable cardioverter defibrillators for 1.5-T magnetic resonance imaging (MRI) scans regardless of whether magnetic resonance-conditional or conventional devices were used.

2. Despite the small study population, our study enrolled variable cases representative of real clinical practice, such as cases of MRI being performed more than twice and performed without mode changes

\section{Conflict of interest}

No potential conflict of interest relevant to this article was reported.

\section{Acknowledgments}

This work was supported by a grant from the Seoul National University Bundang Hospital (SNUBH) Research Fund (no. 02-2014-061).

\section{REFERENCES}

1. Niehaus M, Tebbenjohanns J. Electromagnetic interference in patients with implanted pacemakers or cardioverter-defibrillators. Heart 2001;86:246-248.

2. Quint DJ. Indications for emergent MRI of the central nervous system. JAMA 2000;283:853-855.

3. Kalin R, Stanton MS. Current clinical issues for MRI scanning of pacemaker and defibrillator patients. Pacing Clin Electrophysiol 2005;28:326-328.

4. Hayes DL, Holmes DR Jr, Gray JE. Effect of 1.5 tesla nuclear magnetic resonance imaging scanner on implanted permanent pacemakers. J Am Coll Cardiol 1987;10:782786.

5. Shellock FG, Tkach JA, Ruggieri PM, Masaryk TJ. Cardiac pacemakers, ICDs, and loop recorder: evaluation of translational attraction using conventional ("long-bore") and "short-bore" 1.5- and 3.0-Tesla MR systems. J Cardiovasc Magn Reson 2003;5:387-397.

6. Luechinger R, Zeijlemaker VA, Pedersen EM, et al. In vivo heating of pacemaker leads during magnetic resonance imaging. Eur Heart J 2005;26:376-383.

7. Mollerus M, Albin G, Lipinski M, Lucca J. Ectopy in patients with permanent pacemakers and implantable cardioverter-defibrillators undergoing an MRI scan. Pacing Clin Electrophysiol 2009;32:772-778.

8. Gimbel JR, Kanal E. Can patients with implantable pacemakers safely undergo magnetic resonance imaging? J Am Coll Cardiol 2004;43:1325-1327.

9. Martin ET, Coman JA, Shellock FG, Pulling CC, Fair R, Jenkins K. Magnetic resonance imaging and cardiac pacemaker safety at 1.5-Tesla. J Am Coll Cardiol 2004;43:13151324.

10. Roguin A. Magnetic resonance imaging in patients with implantable cardioverter-defibrillators and pacemakers. J Am Coll Cardiol 2009;54:556-557.

11. European Society of Cardiology (ESC); European Heart Rhythm Association (EHRA), Brignole M, et al. 2013 ESC guidelines on cardiac pacing and cardiac resynchronization therapy: the task force on cardiac pacing and resynchronization therapy of the European Society of Cardiology (ESC). Developed in collaboration with the European Heart Rhythm Association (EHRA). Europace 2013;15:1070-1118.

12. Verma A, Ha AC, Dennie C, et al. Canadian Heart Rhythm Society and Canadian Association of Radiologists consensus statement on magnetic resonance imaging with cardiac implantable electronic devices. Can J Cardiol 2014;30:1131-1141.

13. Maglia G, Curnis A, Brieda M, et al. Assessing access to MRI of patients with magnetic resonance-conditional pacemaker and implantable cardioverter defibrillator systems: the Really ProMRI study design. J Cardiovasc Med (Hagerstown) 2015;16:715-720. 
14. Nazarian S, Roguin A, Zviman MM, et al. Clinical utility and safety of a protocol for noncardiac and cardiac magnetic resonance imaging of patients with permanent pacemakers and implantable-cardioverter defibrillators at 1.5 tesla. Circulation 2006;114:1277-1284.

15. Sommer T, Naehle CP, Yang A, et al. Strategy for safe performance of extrathoracic magnetic resonance imaging at 1.5 tesla in the presence of cardiac pacemakers in non-pacemaker-dependent patients: a prospective study with 115 examinations. Circulation 2006;114:1285-1292.

16. Levine GN, Gomes AS, Arai AE, et al. Safety of magnetic resonance imaging in patients with cardiovascular devices: an American Heart Association scientific statement from the Committee on Diagnostic and Interventional Cardiac Catheterization, Council on Clinical Cardiology, and the Council on Cardiovascular Radiology and Intervention: endorsed by the American College of Cardiology Foundation, the North American Society for Cardiac Imaging, and the Society for Cardiovascular Magnetic Resonance. Circulation 2007;116:2878-2891.

17. Burke PT, Ghanbari H, Alexander PB, Shaw MK, Daccarett M, Machado C. A protocol for patients with cardiovascular implantable devices undergoing magnetic resonance imaging (MRI): should defibrillation threshold testing be performed post-(MRI). J Interv Card Electrophysiol 2010;28:59-66.

18. Forleo GB, Santini L, Della Rocca DG, et al. Safety and efficacy of a new magnetic resonance imaging-compatible pacing system: early results of a prospective comparison with conventional dual-chamber implant outcomes. Heart Rhythm 2010;7:750-754.

19. Wilkoff BL, Bello D, Taborsky M, et al. Magnetic resonance imaging in patients with a pacemaker system de- signed for the magnetic resonance environment. Heart Rhythm 2011;8:65-73.

20. Jung W, Zvereva V, Hajredini B, Jackle S. Safe magnetic resonance image scanning of the pacemaker patient: current technologies and future directions. Europace 2012;14:631-637.

21. Gold MR, Sommer T, Schwitter J, et al. Full-body MRI in patients with an implantable cardioverter-defibrillator: primary results of a randomized study. J Am Coll Cardiol 2015;65:2581-2588.

22. The Task Force on cardiac pacing and resynchronization therapy of the European Society of Cardiology (ESC) Developed in collaboration with the European Heart Rhythm Association (EHRA), Brignole M, Auricchio A, et al. 2013 ESC Guidelines on cardiac pacing and cardiac resynchronization therapy. Rev Esp Cardiol (Engl Ed) 2014;67:58.

23. Roguin A, Zviman MM, Meininger GR, et al. Modern pacemaker and implantable cardioverter/defibrillator systems can be magnetic resonance imaging safe: in vitro and in vivo assessment of safety and function at 1.5 T. Circulation 2004;110:475-482.

24. Nazarian S, Hansford R, Roguin A, et al. A prospective evaluation of a protocol for magnetic resonance imaging of patients with implanted cardiac devices. Ann Intern Med 2011;155:415-424.

25. Russo RJ, Costa HS, Silva PD, et al. Assessing the risks associated with MRI in patients with a pacemaker or defibrillator. N Engl J Med 2017;376:755-764.

26. Hwang YM, Kim J, Lee JH, et al. Cardiac implantable electronic device safety during magnetic resonance imaging. Korean Circ J 2016;46:804-810. 
Supplementary Table 1. Details of the device models and MRI scans used in the present study

\begin{tabular}{|c|c|c|c|c|c|c|c|}
\hline \multirow{2}{*}{$\begin{array}{l}\text { Patient } \\
\text { no. }\end{array}$} & \multirow{2}{*}{ Generator } & \multirow{2}{*}{ Atrial lead } & \multirow{2}{*}{ Ventricular lead } & \multicolumn{2}{|c|}{ Type of device } & \multirow{2}{*}{ MRI site } & \multirow{2}{*}{$\begin{array}{l}\text { MRI } \\
\text { tesla, T }\end{array}$} \\
\hline & & & & Base mode & Change for MR & & \\
\hline$\overline{1}$ & Advisa DR MRI & 5086MRI: $52 \mathrm{~cm}$ & 5086MRI: $58 \mathrm{~cm}$ & DDDR & DDDR & C-spine & 1.5 \\
\hline 2 & Accent DR RF & Isoflex1944: $52 \mathrm{~cm}$ & Isoflex1948: $58 \mathrm{~cm}$ & & DOOR & L-S spine & 1.5 \\
\hline 3 & Advisa DR MRI & 5086MRI: $52 \mathrm{~cm}$ & 5086MRI: $58 \mathrm{~cm}$ & & DOO & L-spine & 1.5 \\
\hline 4 & Altrua 60 & No information & No information & & No information & Brain & 1.5 \\
\hline 5 & Advisa DR MRI & $5072: 52 \mathrm{~cm}$ & $4074: 58 \mathrm{~cm}$ & & DOO & Brain & 1.5 \\
\hline 6 & Advisa DR MRI & $5076: 52 \mathrm{~cm}$ & $5076: 58 \mathrm{~cm}$ & & DOO & L-spine & 1.5 \\
\hline 7 & Advisa DR MRI & 5086MRI: $52 \mathrm{~cm}$ & 5086MRI: $58 \mathrm{~cm}$ & & DOO & Brain & 1.5 \\
\hline 8 & Accent MRI & Tendril MRI: $52 \mathrm{~cm}$ & $\begin{array}{l}\text { Tendril MRI: } 5^{8} \\
\mathrm{~cm}\end{array}$ & & $\mathrm{DOO}$ & Brain & 1.5 \\
\hline 9 & Advisa DR MRI & $5076: 52 \mathrm{~cm}$ & $5076: 58 \mathrm{~cm}$ & & DOO & Brain & 1.5 \\
\hline 10 & Advisa DR MRI & $5076: 52 \mathrm{~cm}$ & $5076: 58 \mathrm{~cm}$ & & DOO & Brain & 1.5 \\
\hline 11 & Advisa DR MRI & 5086MRI: $52 \mathrm{~cm}$ & 5086MRI: $58 \mathrm{~cm}$ & AAIR/DDDR & $\mathrm{DOO}$ & Brain & 1.5 \\
\hline 12 & Advisa DR MRI & 5086MRI: $52 \mathrm{~cm}$ & 5086MRI: $58 \mathrm{~cm}$ & & DOO & Brain & 1.5 \\
\hline 13 & Accent RF & Isoflex1944: $52 \mathrm{~cm}$ & Isoflex1948: $5^{8} \mathrm{~cm}$ & DDD & DOO & Brain & 1.5 \\
\hline 14 & Adapta ADDor & $5076: 52 \mathrm{~cm}$ & 4074: $58 \mathrm{~cm}$ & DDD & DDD & Brain & 1.5 \\
\hline 15 & Adapta ADDor & 4568 & 4092 & & DOO & L-S spine & 1.5 \\
\hline 16 & Advisa DR MRI & 4574 & 4074 & & DOO & C-T-L spine & 1.5 \\
\hline 17 & Adapta ADDor & 4568 & 4092 & & POR/DDD & L-spine & 1.5 \\
\hline 18 & Accent MRI & Tendril MRI: $52 \mathrm{~cm}$ & $\begin{array}{l}\text { Tendril MRI: } 58 \\
\text { cm }\end{array}$ & & DOO & Brain & 1.5 \\
\hline 19 & Accent RF & $\begin{array}{l}\text { Tendril STS 2088TC: } \\
52 \mathrm{~cm}\end{array}$ & Isoflex1948: $58 \mathrm{~cm}$ & DDD & VOO & Brain & 1.5 \\
\hline 20 & Adapta ADDo1 & $5076: 52 \mathrm{~cm}$ & 4074: $58 \mathrm{~cm}$ & DDD/AAI & DOO & Knee & 1.5 \\
\hline 21 & Regency SC 2402L & & Isoflex1948: $58 \mathrm{~cm}$ & & VOO & Brain & 1.5 \\
\hline 22 & Sigma SS & & $4074: 58 \mathrm{~cm}$ & & VOO & Brain & 1.5 \\
\hline 23 & $\begin{array}{l}\text { Identity ADx XL } \\
\text { DR } 5386\end{array}$ & 1642T: $52 \mathrm{~cm}$ & 1646T: $58 \mathrm{~cm}$ & & VOO & L-spine & 1.5 \\
\hline 24 & $\begin{array}{l}\text { Sustain XL SC PM } \\
1134\end{array}$ & & $\begin{array}{l}\text { Biotronics: no } \\
\text { information }\end{array}$ & & VOO & Brain & 1.5 \\
\hline 25 & Accent MRI & & LPA 1200M: $58 \mathrm{~cm}$ & VVIR & VOO & L-S spine & 1.5 \\
\hline 26 & Enpulse E2SRo3 & & $4074: 58 \mathrm{~cm}$ & & VOO & Brain & 1.5 \\
\hline 27 & Enpulse E2SRo3 & & $4074: 58 \mathrm{~cm}$ & & VOO & Brain & 1.5 \\
\hline 28 & Regency SR+ & & 3650 & & VOO & $\begin{array}{l}\text { Intra-auditory } \\
\text { canal }\end{array}$ & 1.5 \\
\hline 29 & Fortify VR & & $\begin{array}{l}\text { Durata } 7170 Q: 58 \\
\mathrm{~cm}\end{array}$ & ICD & VOO & L-spine & 1.5 \\
\hline 30 & Evera XT VR & & 6935M: $62 \mathrm{~cm}$ & ICD & OVO & Brain & 1.5 \\
\hline 31 & Atlas VR V-193 & & Riata 1570 & ICD & Pacing off & $\begin{array}{l}\text { C-spine and } \\
\text { L-spine }\end{array}$ & 1.5 \\
\hline 32 & Fortify VR & & $\begin{array}{l}\text { Durata } 7170 Q: 5^{8} \\
\mathrm{~cm}\end{array}$ & ICD & VOO & Pelvic & 1.5 \\
\hline 33 & Evera XT VR & & 6935M: $62 \mathrm{~cm}$ & ICD & VOO & C-spine & 1.5 \\
\hline 34 & Fortify VR & & $\begin{array}{l}\text { Duarata 7170Q: } 58 \\
\mathrm{~cm}\end{array}$ & ICD & VOO & Brain & 1.5 \\
\hline 35 & Fortify DR & $\begin{array}{l}\text { Tendril STS } \\
\text { 2088TC: } 52 \mathrm{~cm}\end{array}$ & $\begin{array}{l}\text { Duarata 7170Q: } \\
58 \mathrm{~cm}\end{array}$ & ICD & VOO & Pelvic & 1.5 \\
\hline
\end{tabular}

MRI, magnetic resonance imaging; MR, magnetic resonance; DDDR, rate response dual chamber atrioventricular synchronous pacing; C, cervical; DOOR, rate response dual-chamber asynchronous pacing; L, lumbar; DDD, dual chamber atrioventricular synchronous pacing; AAIR, rate response atrial inhibited pacing; T, thoracic; POR, power on reset; VOO, ventricular asynchronous pacing; AAI, atrial inhibited pacing; VVIR, rate response ventricular inhibited pacing; ICD, implantable cardioverter defibrillator; OVO, no pacing, sensing only mode. 
Han $D$, et al. MRI in patients with pacemakers or ICDs

Supplementary Table 2. Median values of the atrial and ventricular impedance, lead sensing, and captured threshold among pre-/post-MRI and follow-up

\begin{tabular}{lcccc}
\hline & Pre-MRI & Post-MRI & Follow-up & p value \\
\hline Impedance, $\Omega$ & & & & \\
Atrial & 475 & 460 & 518 & 0.99 \\
Ventricular & 575 & 536.5 & 556 & 0.79 \\
Sensing, mV & & & & \\
Atrial & 2.8 & 2.5 & 2.9 & 0.92 \\
Ventricular & 11.2 & 12 & 9.75 & 0.72 \\
Capture threshold, V & & & & \\
Atrial & 0.5 & 0.5 & 0.5 & 0.82 \\
\hline Ventricular & 0.75 & 0.75 & 0.75 & 0.79 \\
\hline
\end{tabular}

MRI, magnetic resonance imaging. 
Supplementary Table 3. Differences in the atrial and ventricular impedance, lead sensing, and captured threshold values between the MR-conditional and conventional groups at pre-/post-MRI and follow-up

\begin{tabular}{|c|c|c|c|}
\hline & MR-conditional $(\mathrm{n}=16)$ & Conventional $(\mathrm{n}=27)$ & $p$ value \\
\hline \multicolumn{4}{|l|}{ Impedance, $\Omega$} \\
\hline \multicolumn{4}{|l|}{ Atrial } \\
\hline Pre-MRI & $466.4 \pm 49.7$ & $444.1 \pm 66.3$ & 0.35 \\
\hline Post-MRI & $463.8 \pm 44.4$ & $449 \cdot 3 \pm 61.4$ & 0.52 \\
\hline Follow-up & $458.5 \pm 45 \cdot 3$ & $465.7 \pm 58.9$ & 0.77 \\
\hline \multicolumn{4}{|l|}{ Ventricular } \\
\hline Pre-MRI & $539.1 \pm 73.8$ & $587.4 \pm 139.2$ & 0.22 \\
\hline Post-MRI & $542.4 \pm 72.9$ & $586.1 \pm 135 \cdot 9$ & 0.24 \\
\hline Follow-up & $543.6 \pm 79.1$ & $556.5 \pm 102.3$ & 0.72 \\
\hline \multicolumn{4}{|l|}{ Sensing, $\mathrm{mV}$} \\
\hline \multicolumn{4}{|l|}{ Atrial } \\
\hline Pre-MRI & $2.24 \pm 1.18$ & $2.48 \pm 1.09$ & 0.67 \\
\hline Post-MRI & $2.19 \pm 1.12$ & $2.87 \pm 1.59$ & 0.31 \\
\hline Follow-up & $1.78 \pm 0.94$ & $2.84 \pm 1.93$ & 0.15 \\
\hline \multicolumn{4}{|l|}{ Ventricular } \\
\hline Pre-MRI & $11.1 \pm 3.88$ & $12.6 \pm 5.86$ & 0.39 \\
\hline Post-MRI & $11.4 \pm 3.89$ & $12.9 \pm 5.82$ & 0.36 \\
\hline Follow-up & $12.5 \pm 4.47$ & $10.7 \pm 5.65$ & 0.33 \\
\hline \multicolumn{4}{|c|}{ Capture threshold, V } \\
\hline \multicolumn{4}{|l|}{ Atrial } \\
\hline Pre-MRI & $0.53 \pm 0.15$ & $0.71 \pm 0.29$ & 0.08 \\
\hline Post-MRI & $0.53 \pm 0.13$ & $0.54 \pm 0.18$ & 0.89 \\
\hline Follow-up & $0.5 \pm 0$ & $0.74 \pm 0.36$ & 0.05 \\
\hline \multicolumn{4}{|l|}{ Ventricular } \\
\hline Pre-MRI & $0.71 \pm 0.23$ & $0.68 \pm 0.27$ & 0.67 \\
\hline Post-MRI & $0.73 \pm 0.16$ & $0.73 \pm 0.26$ & 0.96 \\
\hline Follow-up & $0.76 \pm 0.24$ & $0.71 \pm 0.24$ & 0.53 \\
\hline
\end{tabular}

Values are presented as mean $\pm \mathrm{SD}$.

MR, magnetic resonance; MRI, magnetic resonance imaging. 
Han D, et al. MRI in patients with pacemakers or ICDs

Supplementary Table 4. Initial manufacturing dates of the generators used and the manufacturing dates of the study patients' specific generators.

\begin{tabular}{|c|c|c|c|c|}
\hline & & Generator & Initial manufacturing date & $\begin{array}{c}\text { The earliest patients' generator } \\
\text { manufacturing date }\end{array}$ \\
\hline \multirow[t]{10}{*}{$\mathrm{PM}$} & Medtronics & Adapta DDD ${ }^{\mathrm{a}}$ & 28-Nov-2005 & 5-Apr-2013 \\
\hline & & Sigma SS & 25-Jan-1999 & 19-Feb-2007 \\
\hline & & EnPulse SSIR ${ }^{b}$ & 14-Apr-2003 & 23-Jun-2008 \\
\hline & & Kappa KD甲o3 & 22-Nov-2001 & 26-Mar-2004 \\
\hline & St. Jude & Regency SR + 2400L $\mathrm{L}^{\mathrm{c}}$ & 1995 & 20-Jan-2009 \\
\hline & & Identity ADx XL DR 5386 & 2003 & 17-Nov-2006 \\
\hline & & Sustain XL SC PM1134 & 2011 & 11-Jun-2013 \\
\hline & & Accent RF ${ }^{\mathrm{d}}$ & Jul-2009 & 7-Jul-2011 \\
\hline & Biotronik & Effecta $\mathrm{S}$ & Feb-2011 & Jul-2012 \\
\hline & Boston scientific & Altrua $60 \mathrm{~S} 603$ & 15-May-2008 & 25-Jun-2010 \\
\hline \multirow[t]{4}{*}{$\mathrm{ICD}$} & St. Jude & Atlas VR V-193 & 2003 & o1-Mar-2008 \\
\hline & & Fortify $\mathrm{VR}^{\mathrm{e}}$ & May-2010 & 9-Mar-2012 \\
\hline & & Fortify DR & May-2010 & 9-Aug-2011 \\
\hline & Medtronics & Evera XT VR ${ }^{\mathrm{f}}$ & Apr-2012 & 8-Oct-2013 \\
\hline
\end{tabular}

PM, pacemaker; ICD, implantable cardioverter defibrillator.

$\mathrm{a}, \mathrm{b}, \mathrm{c}, \mathrm{d}, \mathrm{e}, \mathrm{f}$ In cases of patients with these devices, we documented the earliest date when the patients' generators had been manufactured. 


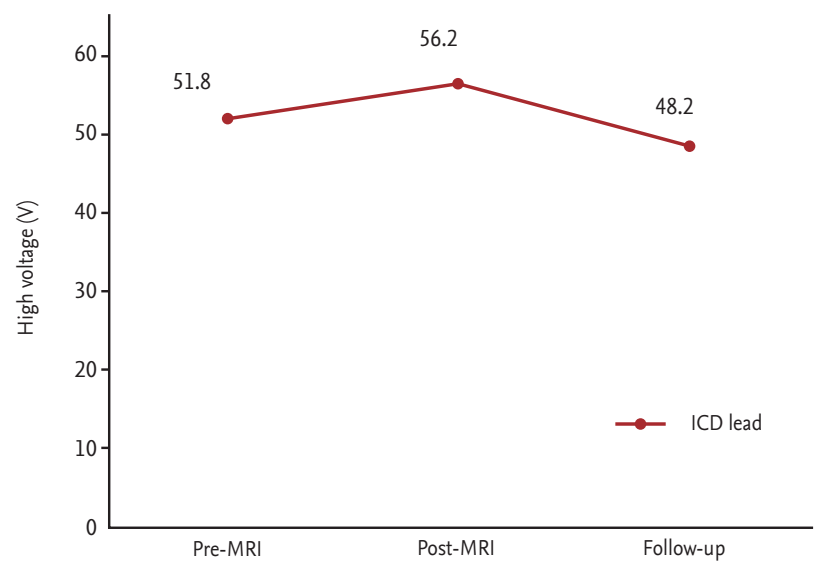

Supplementary Figure 1. Changes in the ventricular high voltage of the implantable cardioverter defibrillator (ICD) lead at pre-/post-magnetic resonance imaging (MRI) and follow-up visit $(p=0.79)$. All ICDs were conventional devices. 


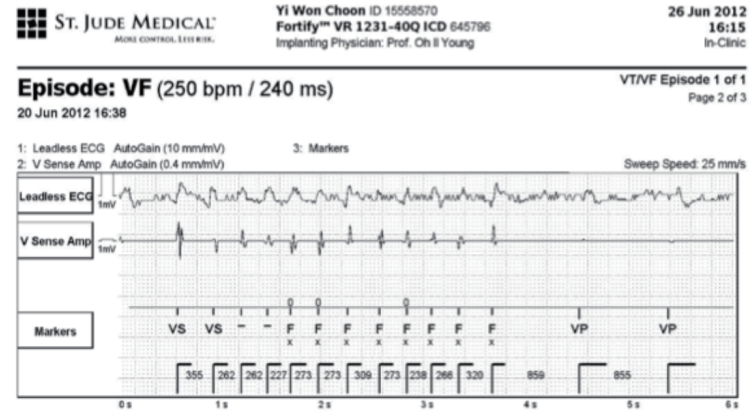

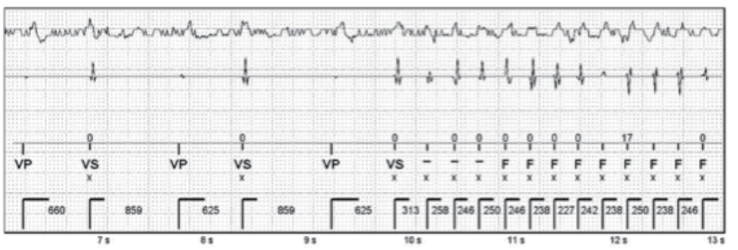

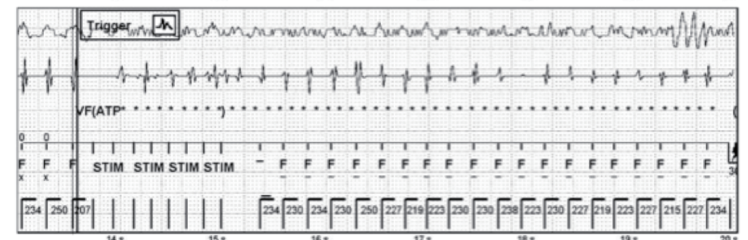

Forrty" VR 1231-400 ICD (645796 prc.0.95) Bundang Seou Natonal Umerty hosp

VTNF Episode 1 of 1 Page 2 of 3
$26 \operatorname{lin} 201216: 15$

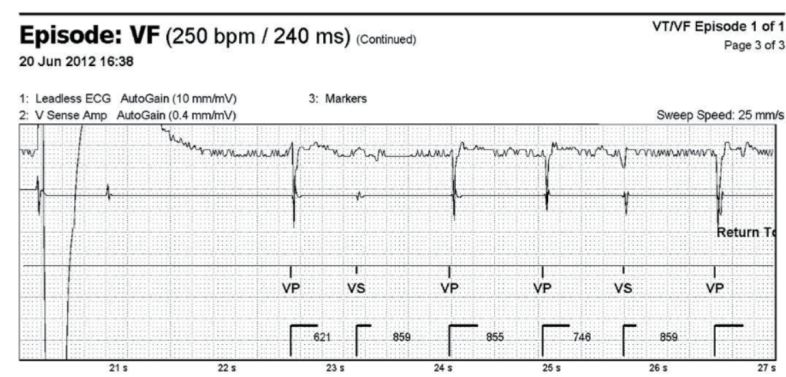

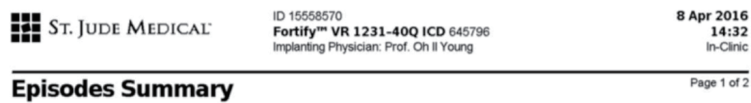
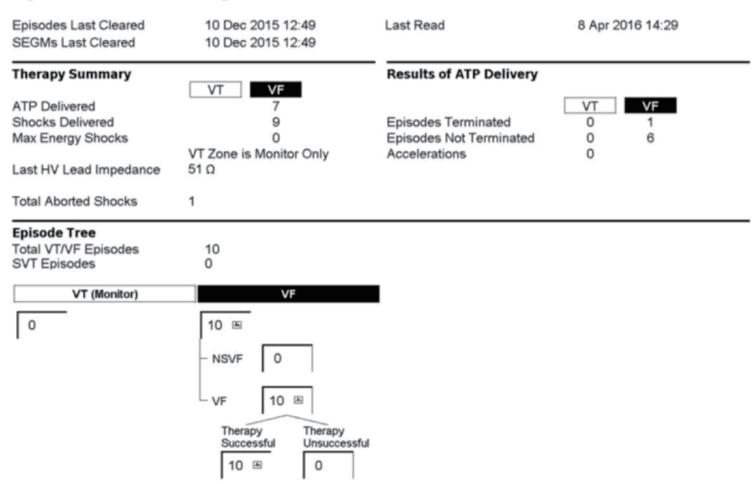

\begin{tabular}{|c|c|c|c|c|c|}
\hline \multicolumn{6}{|l|}{ VT/VF Episodes } \\
\hline 11 Mar $20168: 55$ & VF & 255 & $00: 15$ & $30 \mathrm{~s}$ & \\
\hline 10 Mar 2016 1:57 & & 230 & & & \\
\hline $9 \operatorname{Mar} 201617: 13$ & VF & & $00: 15$ & ATP 301 & \\
\hline 7 Mar $201620: 58$ & vF & 230 & $00: 16$ & ATP, $30 \mathrm{~J}$ & \\
\hline 4 Mar 201620.44 & VF & 235 & $00: 18$ & ATP, $30 \mathrm{~s}$ & \\
\hline 4 Mar 20167:36 & VF & 226 & 00:15 & ATP, $30 \mathrm{~J}$ & \\
\hline $29 \mathrm{Feb} 2016$ 14:21 & VF & 235 & 00:15 & ATP, $30 \mathrm{~J}$ & \\
\hline $22 \mathrm{Feb} 20161: 36$ & VF & 260 & 00:16 & $30 \mathrm{~s}$ & \\
\hline $\begin{array}{l}14 \operatorname{Jan} 20164: 12 \\
7 \mathrm{~J} a n \\
2016 \\
16: 18\end{array}$ & $\begin{array}{l}\text { VF } \\
\text { VF }\end{array}$ & $\begin{array}{l}{ }_{250}^{250} \\
285\end{array}$ & $\begin{array}{l}00: 07 \\
00: 15\end{array}$ & ${ }_{30 \mathrm{~N}}^{\text {ATP }}$ & \\
\hline $\begin{array}{l}\text { Other Episodes } \\
\text { Date / Time }\end{array}$ & Type & & Peak A Rate & Duration & Alerts \\
\hline 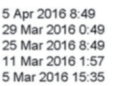 & $\begin{array}{l}\text { Morphology Template Update } \\
\text { Morphology Template Update } \\
\text { Morphology Template Udate } \\
\text { Morphology Template Udatete } \\
\text { Morphology Template Udatate }\end{array}$ & & & & \\
\hline
\end{tabular}

Fortity" VR 1231-400 ICD (645796 prC.D.95) S.N.U.B.H

Episodes Summary Page 1 of 2
8 Apr 201614.32

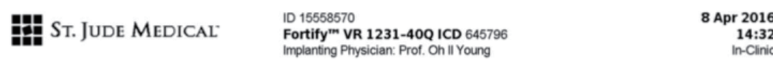

Episode: VF (230 $\left.\mathrm{min}^{-1} / 260 \mathrm{~ms}\right) \quad$ VTNF Episode 9 of 10

\section{Mar 2016 1:57}

$\begin{array}{lll}\text { 1: Leadess ECG AutoGain (10 mmmn) } & \text { 3: Markers }\end{array}$ Sweep Speed. $25 \mathrm{~mm}$
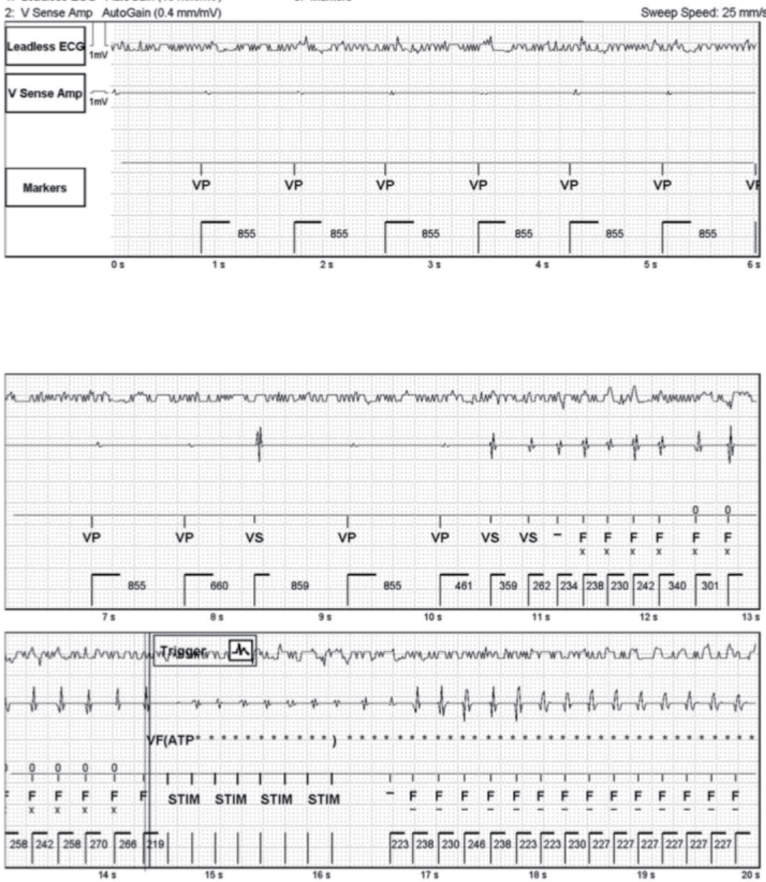

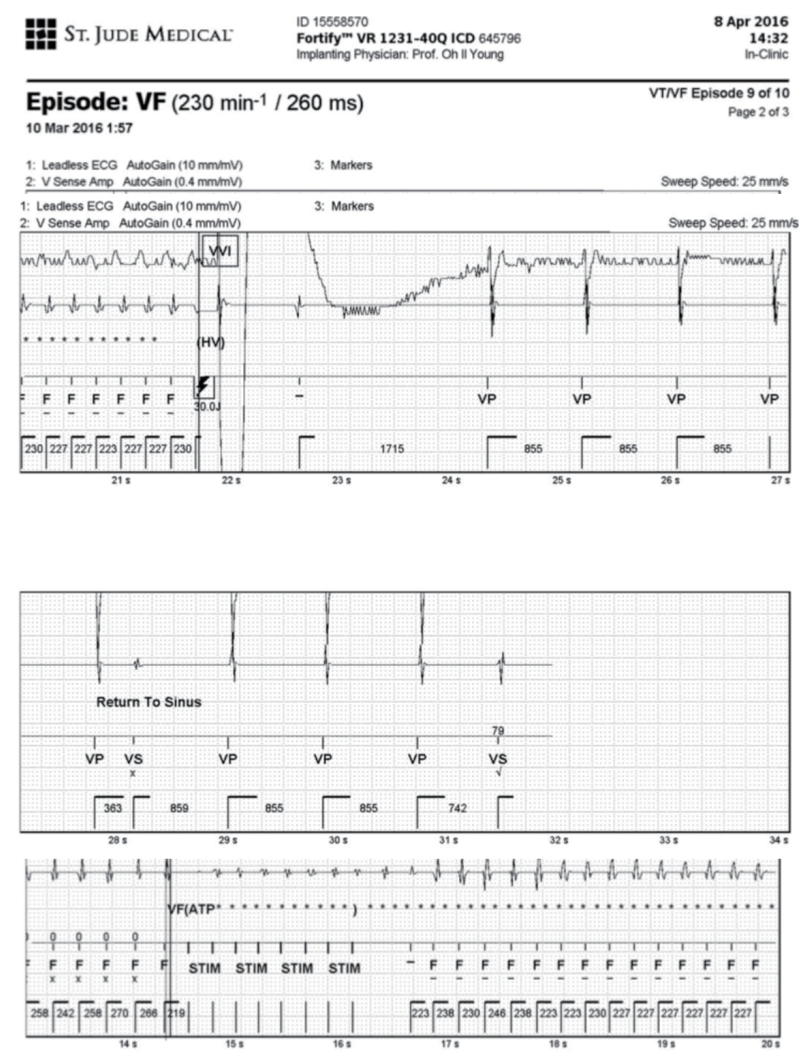

Supplementary Figure 2. Electrogram findings of a patient in whom therapeutic shock was delivered. The implantable cardioverter (ICD) worked normally before and after the magnetic resonance (MRI) scan. This patient had vascular dementia, stroke, end-stage renal disease treated by hemodialysis, heart failure due to ischemic cardiomyopathy, and arteriosclerosis obliterans. He had an ICD due to ischemic cardiomyopathy. The patient experienced therapeutic shock on June 2012, without any symptoms such as palpitation, chest discomfort, and dizziness. After the event, there were no reports or records of ventricular tachycardia/ventricular fibrillation (VT/VF) for 3 years. Three years later, the patient's dementia and arteriosclerosis obliterans of the left leg worsened and he became bedridden, after which a coccyx sore developed. He was repeatedly admitted for the treatment of fever, pneumonia, and the coccyx sore. (A) The patient had already experienced one episode of therapeutic shock in June 2012, before the MRI scan. The electrogram at this time showed appropriate shock delivery. (B) The patient underwent MRI scanning to evaluate the extent of his coccyx sore on December 2015. He experienced 10 episodes of spontaneous VT/VF and nine therapeutic shocks after MRI. All therapeutic shocks were appropriate. The red box shows the days of the events and therapeutic shock delivery. 